\title{
Turbulent Rayleigh-Bénard convection scaling in a vertical channel using the lattice Boltzmann method
}

\author{
Y. Wei \& H. Dou \\ Faculty of Mechanical Engineering and Automation, \\ Zhejiang Sci-Tech University, China
}

\begin{abstract}
The existing scaling laws for turbulent Rayleigh-Bénard convection of air in a differentially heated vertical channel is investigated via the lattice Boltzmann method (LBM). Based on the arguments of an inner (near-wall) and outer (channel centre) region, the simulation results are found to support a minus onethird power law for the mean temperature in an overlap region. Using the inner and outer temperature profiles, an implicit heat transfer equation is shown. A correction term is non-negligible for the present $R a$ range when compared with the explicit equations found in this paper. Finally, it is shown that the results of present LBM about the Nusselt number $N u$ as a function of Rayleigh number are in good agreement with the theoretical values.

Keywords: turbulent, Rayleigh-Bénard convection, scaling, LBM.
\end{abstract}

\section{Introduction}

The heat transfer in a channel has been widely studied in some thermal engineering applications such as solar thermal receivers. One of the prevalent industry practices is to control the heat gain or loss of the building by insulating the external building envelope. The key to a deeper understanding of the mechanisms of heat and momentum in turbulent Rayleigh-Bénard convection lies in better access to the dynamic in the scaling of temperature and velocity of an inner (near-bottom wall) and outer (channel centre) region [1]. The Reynoldsaveraged Navier-Stokes simulations (RANS) and the large eddy simulations (LES) are important to have accurate wall models, especially since these models set the boundary conditions for calculations close to the wall [2]. The added 
computational cost associated with fine grid spacing, a requirement for capturing rapid changes in flow physics near the wall are reduced. As such, it is important to have accurate wall models that are valid for a large range of flow conditions. Rayleigh-Bénard convection, as a typical benchmark problem, has been received noticeable attention from the lattice Boltzmann method (LBM) community owing to its geometric simplicity and its complex flow features at different Rayleigh numbers. Although most of studies just concentrate on the laminar and transitional Rayleigh-Bénard convection [3, 4].

Hölling and Herwig and Shiri and George have developed wall functions using various scaling analyses (e.g. [5-8]). However, the opinions of these studies have largely been found to differ, possibly due to the limited numerical data at the time. The existing scaling laws for turbulent Rayleigh-Bénard convection of air in a differentially heated vertical channel are studied in this paper. Based on the arguments of an inner (near-wall) and outer (channel centre) region, the results of simulation are found to support a minus one-third power law for the mean temperature in an overlap region. Using the inner and outer temperature profiles, an implicit heat transfer equation is shown. In the following, a simple lattice Boltzmann thermal model will be briefly described at first. Then the problem definition and numerical verification are followed. After that, the detailed discussions of turbulence Rayleigh-Bénard convection scaling are investigated. Finally, some concluding remarks are provided.

\section{Numerical method}

The numerical algorithm used is based on the discrete kinetic models. The starting point is a standard coupled mesoscopic dynamics described by [4]:

$$
\begin{gathered}
f_{i}\left(x+c_{i} \Delta t, t+\Delta t\right)=f_{i}(x, t)+\left[f_{i}^{e q}(x, t)-f_{i}(x, t)\right] / \tau_{v}+F_{i} \\
g_{i}\left(x+c_{i} \Delta t, t+\Delta t\right)=g_{i}(x, t)+\left[\mathrm{g}_{i}^{e q}(x, t)-g_{i}(x, t)\right] / \tau_{T}
\end{gathered}
$$

where $f_{i}(x, t), g_{i}(x, t)$ stand for the probability density functions to find at $(x, t)$ a particle velocity belongs to a discrete and limited set $c_{i}$ (with $i=0,1,2, \cdots 18$ in the $D 3 Q 19 \mathrm{LBM}$ adopted here [3]). $F_{i}$ is the momentum input from the buoyant body force, $\tau_{v}$ and $\tau_{T}$ are the relaxation time for flow and temperature LB equations. The additional buoyant body force term $F_{i}$ can be formulated by the Boussinesq approximation, i.e.

$$
F_{i}=3 w_{i} \cdot \rho \cdot \mathbf{g} \cdot \beta \cdot T \cdot c_{i y}
$$

where $\mathbf{g}$ is the acceleration of gravity, $\beta$ is the thermal expansion coefficient, $c_{i y}$ is the y-component of $c_{i} \cdot f_{i}^{e q}$ is a single-particle equilibrium distribution 
function which is derived from a Maxwell-Boltzmann distribution and can be approximated to

$$
\left.f_{i}^{e q}=\rho w_{i}\left[1+\frac{\boldsymbol{c}_{i} \cdot \boldsymbol{u}}{c_{s}^{2}}+\frac{\left(\boldsymbol{c}_{i} \cdot \boldsymbol{u}\right)^{2}}{c_{s}^{2}}-\frac{u^{2}}{2 c_{s}^{2}}\right] g_{i}^{e q}=w_{i} T\left[1+\frac{\left(\mathbf{c}_{i} \cdot \mathbf{u}\right)}{c_{s}^{2}}\right)\right]
$$

For a D3Q19 lattice, shown in Figure 1, we have:

$$
w_{i}=\left\{\begin{aligned}
1 / 3, & i=0 \\
1 / 18, & i=1,2, \ldots, 6 \\
1 / 36, & i=7,8, \ldots, 18
\end{aligned}\right.
$$

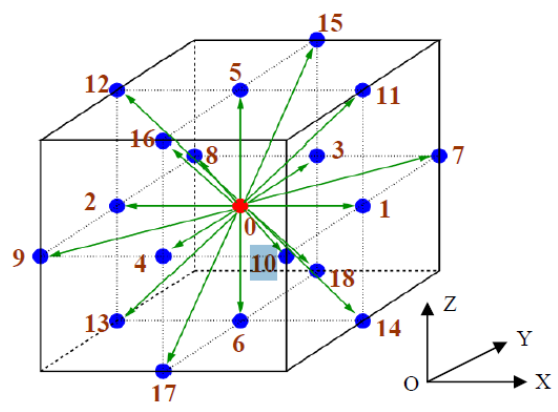

Figure 1: $\quad$ Lattice velocity directions in D3Q19 lattice structures [3].

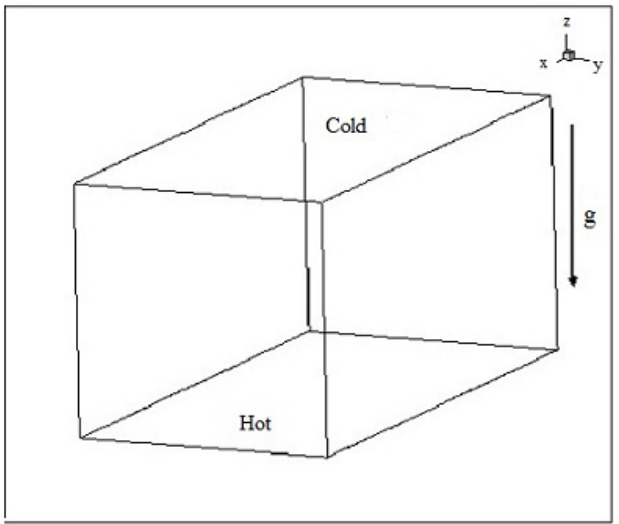

Figure 2: Schematic diagram of RB convection in a vertical channel.

A Chapman-Enskog expansion leads to the equations (5)-(7) for the density, momentum, and temperature equation based on equations (1) and (2). The streaming step on the left-hand side reproduces the inertial terms in the hydrodynamical equations, whereas dissipation and thermal diffusion are 
connected to the relaxation (towards equilibrium) properties in the right hand side, with $\tau_{v}$ and $\tau_{T}$ related to the relaxation times.

$$
\begin{gathered}
\frac{\partial \rho}{\partial t}+\nabla \cdot(\rho \mathbf{u})=0 \\
\frac{\partial(\rho \mathbf{u})}{\partial t}+\nabla \cdot(\rho \mathbf{u u})=-\nabla p+\nabla \cdot(2 \rho \nu \mathbf{S})-g \beta \Delta T \\
\frac{\partial T}{\partial t}+\mathbf{u} \cdot \nabla T=\Gamma \nabla^{2} T
\end{gathered}
$$

\section{Numerical simulations and discussions}

A computational domain size is defined by $L_{x} \times L_{y} \times L_{z}$ with the resolutions $M_{x} \times M_{y} \times M_{z}=456 \times 228 \times 228$ for $R a=6.0 \times 10^{6}$, and $M_{x} \times M_{y} \times M_{z}=656 \times 328 \times 328$ for $R a=4.0 \times 10^{7}$. The side boundaries of the channel were assigned periodic boundary conditions for both the flow and temperature fields. Meanwhile, the upper and bottom solid boundaries were assigned no-slip conditions for the flow field and were assigned non-dimensional temperatures of $T_{u p}=0$ and $T_{\text {down }}=1$.

Figure 3 presents snapshots of the isosurfaces of the temperature fields. The red is hot plumes and the blue is cold plumes. By integrating the Eq. (7), we obtain

$$
\Gamma \frac{\partial T}{\partial z}-u_{z} T=-\frac{q_{z}}{\rho C_{p}}=Q_{z}
$$

which describes a characteristic heat flux constant $Q_{z}$ equivalent to the wall heat flux $q_{z}$ flowing from left to right divided by density $\rho$ and specific heat $C_{p}$. The outer temperature scale depends on the channel half-high $h$, the inner and outer temperature scales are:

$$
T_{i}=\left(\frac{\left|Q_{z}\right|^{3}}{g \beta \Gamma}\right)^{1 / 4}, T_{o}=\left(\frac{\left|Q_{z}\right|^{2}}{g \beta h}\right)^{1 / 3}
$$

The respective inner and outer length scales are defined as:

$$
l_{i}=\left(\frac{\Gamma^{3}}{g \beta\left|q_{z}\right|}\right)^{1 / 4} \text { and } l_{o}=h
$$

To use the above scaling and the gradient-matching approach, the following power-law forms of the temperature wall functions are obtained [7].

$$
\frac{T_{h}-\bar{T}}{T_{i}}=-c_{1}\left(\frac{z}{l_{i}}\right)^{-1 / 3}-c_{2}(\operatorname{Pr}) \text {, and } \frac{\bar{T}-T_{r e f}}{T_{o}}=c_{1}\left(\frac{z}{l_{i}}\right)^{-1 / 3}-c_{3}
$$




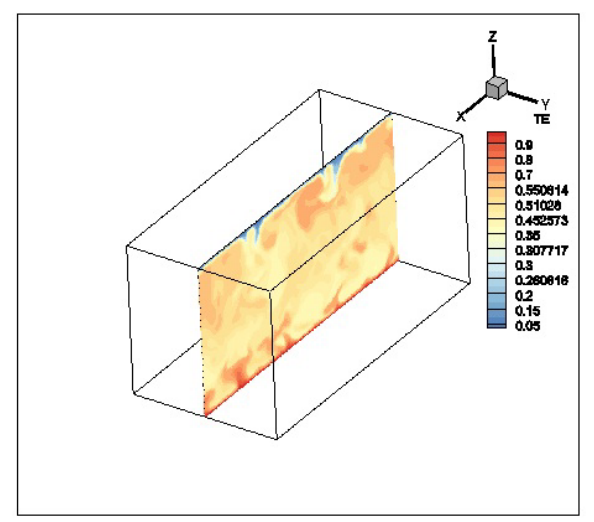

Figure 3: Snapshots of instantaneous isotherms with $M_{y}=L_{y} / 2$ at $R a=4.0 \times 10^{7}$.

The constants to be: $c_{1}=4.2$ and $c_{2}=-5$ are obtained in the study by Versteegh and Nieuwstadt (1999).

In this paper, a new inner temperature wall function with fitted constants is used:

$$
\frac{T_{h}-\bar{T}}{T_{i}}=-4.2\left(\frac{z}{l_{i}}\right)^{-1 / 3}+5.0
$$

Figure 4 shows that temperature data plotted in inner and outer scaling with evidence of adherence to power-law behavior with two Rayleigh numbers. It is found that the LBM results are in good agreement with the present theoretical values. To appraise these wall functions, we qualitatively assess their respective fits to the present data of LBM in both inner and outer scales, shown in Figure 4(a) and (b).

The analysis of the mean heat transport is represented by the Nusselt number in the following section. Firstly we discuss the relations of the turbulent heat flux in their dimensionless form, the Nusselt number $N u=1+\left\langle u_{z} T\right\rangle /(\Gamma \Delta T / H)$ and the Rayleigh number is $R a=\left(\beta \Delta T g H^{3}\right) /(v \Gamma)$. It is shown that the Nusselt number $N u$ is as a function of Rayleigh number. A clear scaling can be seen for $N u(R a)$ for $R a \approx 10^{5}$ to $10^{8}$. The theoretical values in Figure 5 is presented by the following equation $\mathrm{Nu} \sim \mathrm{Ra}^{0.291}$.

Figure 5 displays that the blue dots are the results of present LBM, the black squares represent the simulation results of DNS study by Kaczorowski and Wagner [9], and the red line represents the theoretical values [9]. It can be seen clearly that the results of present LBM are better agreement with the theoretical values than the results of DNS. In addition, it is shown that the results of present LBM about the Nusselt number $N u$ as a function of Rayleigh number are in good agreement with the theoretical values. 

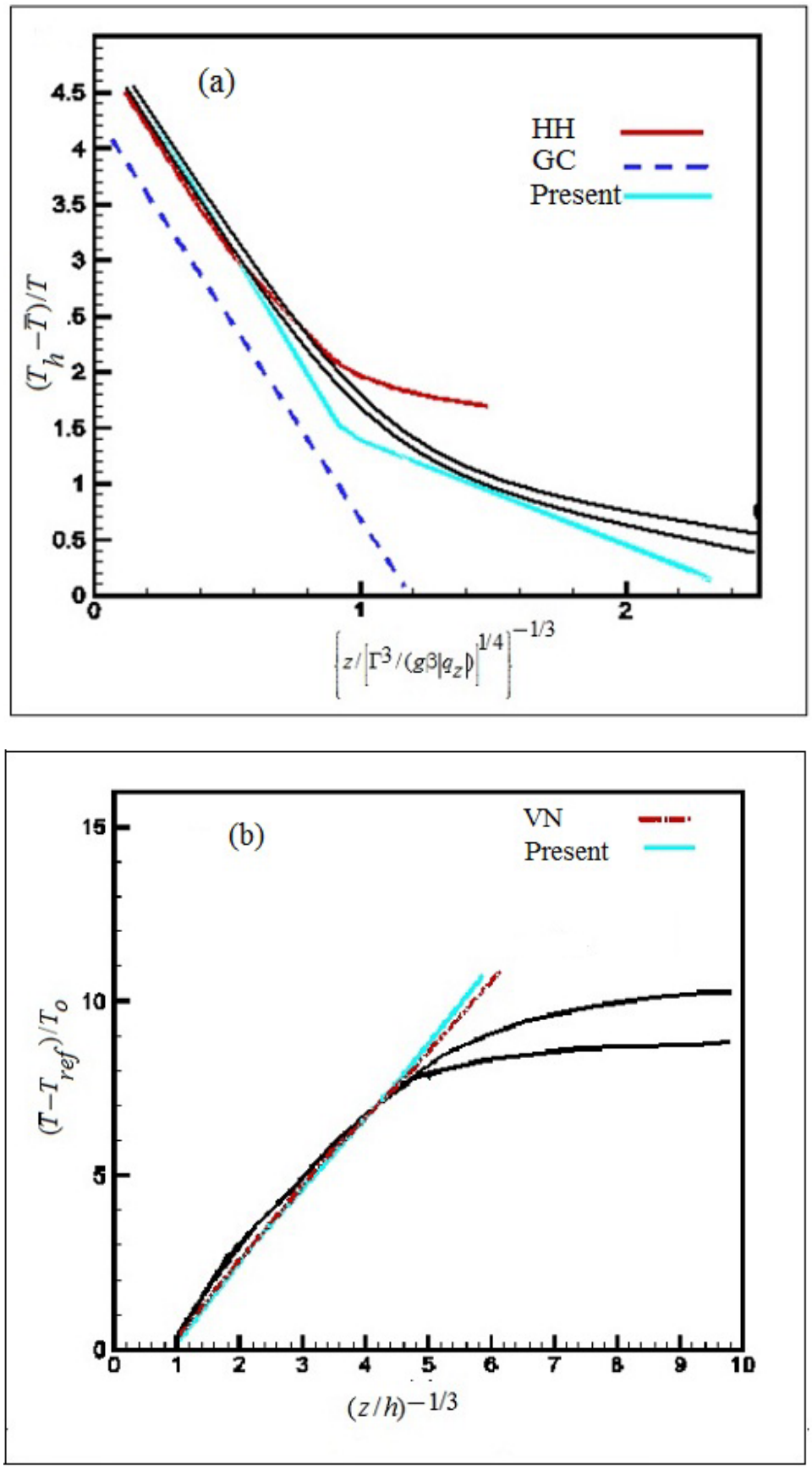

Figure 4: Temperature data plotted in inner and outer scaling with evidence of adherence to power-law behavior. 


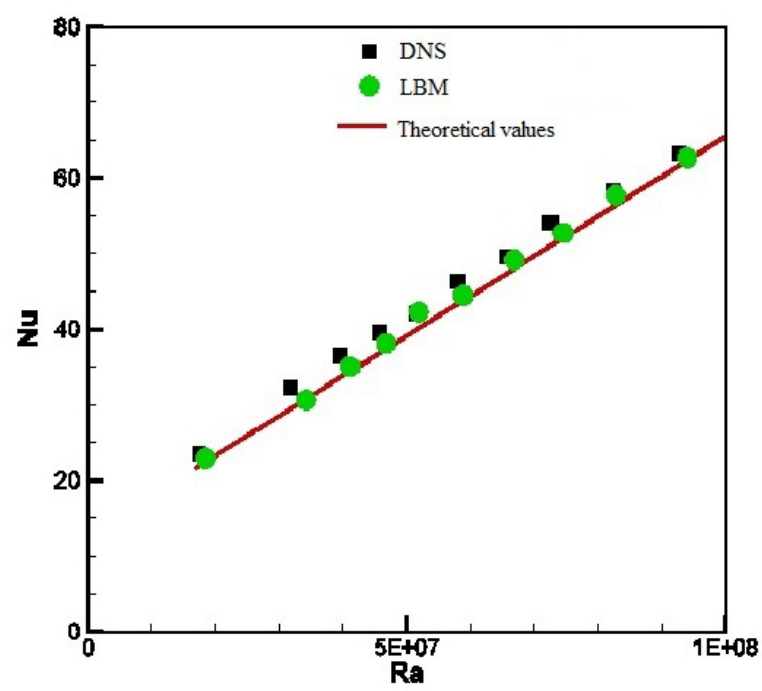

Figure 5: The Nusselt number $N u$ as a function of Rayleigh number.

\section{Conclusion}

We show evidence that provide additional support for a minus one-third power law for the mean inner and outer temperature wall function in an overlap region from the present LBM dataset for $R a=6.0 \times 10^{6}$ and $R a=4.0 \times 10^{7}$. Using the inner and outer temperature profiles, an implicit heat transfer equation is shown. In addition, it is shown that the results of present LBM are better agreement with the experimental results than the results of DNS about the Nusselt number $N u$ as a function of Rayleigh number. Finally, it is shown that the results of present LBM about the Nusselt number $N u$ as a function of Rayleigh number are in good agree with the theoretical vales.

\section{Acknowledgement}

This work is supported by the Science Foundation of Zhejiang Sci-Tech University under Grant No 1130032611308.

\section{References}

[1] N. Azwadi, A.R.M. Rosdzimin, Simulation of natural convection heat transfer in an enclosure using lattice Boltzmann method, J Mekanikal, 2008, 27, 42-50.

[2] M.A. Mussa, S. Abdullah, C.S. Nor Azwadi, N. Muhamad, Simulation of natural convection heat transfer in an enclosure by the lattice-Boltzmann method, Computers \& Fluids, 2011, 44, 162-168. 
[3] Y.H. Qian, D. d'Humieres, P. Lallemand, Lattice BGK models for NavierStokes equation, Europhys Lett, 1992, 17(6), 479-484.

[4] P.H. Kao, R.J. Yang, Simulating oscillatory flows in Rayleigh-Bénard convection using the lattice Boltzmann method, Int J Heat Mass Transfer, 2007, 50, 3315-3328.

[5] C.K. Aidun, Lattice-Boltzmann method for complex flows, Annual Review of Fluid Mechanics, 2010, 42, 439-472.

[6] R.D. Moser, J. Kim, N.N. Mansour, Direct numerical simulation of turbulent channel flow up to Res $=590$, Phys Fluids, 1999, 11, 943-945.

[7] J. Pallares, A. Vernet, Turbulent large-scale structures in natural convection vertical channel flow, Int J Heat Mass Transfer, 2010, 53, 4168-4175.

[8] A. Shiri, W.K. George, Turbulent natural convection in a differentially heated vertical channel. In: Proceedings of 2008 ASME Summer Heat Transfer Conference.

[9] M. Kaczorowski, C. Wagner, Analysis of the thermal plumes in turbulent Rayleigh-Bénard convection based on well resolved numerical simulations, Journal of Fluid Mechanics, 2009, 618, 89-112. 